\title{
El funcionamiento parental y la percepción de apoyo de los estudiantes con diversidad funcional.
}

\section{Parental functioning and the perceived support of students with functional diversity}

\section{Funcionamiento parental - percepción de apoyo}

\author{
Hernán Cedeño Cedeño ${ }^{(\mathbf{1})}$ \\ Inger Maitta Rosado ${ }^{(2)}$ \\ Kerly Sabando Reyes ${ }^{(3)}$ \\ (1) Universidad Técnica de Manabí. e-mail: hernanandrescedeno@gmail.com \\ (2) Universidad Técnica de Manabí, e-mail: ingermaitta7@gmail.com \\ (3) Egresada de Psicología Clínica. e-mail: kerlysabando55@gmail.com
}

Contacto: kerlysabando55@gmail.com

\section{Resumen}

La presente investigación cuantitativa de alcance descriptivo, tiene como objetivo analizar la relación del funcionamiento parental, ejercido por familiares directos o personas no pertenecientes al círculo familiar y la percepción de apoyo de los estudiantes con diversidad funcional de las diferentes escuelas de la Universidad Técnica de Manabí. Para el estudio del funcionamiento parental, se utilizó la escala de funcionamiento parental, dirigida a 43 padres de familia y para conocer la percepción de apoyo, se aplicó una entrevista a 74 estudiantes con diversidad funcional. Como resultado de la investigación se obtuvo que, dentro de estas familias se mantiene un buen funcionamiento parental, por lo cual se destaca la buena comunicación e interacción y vínculos afectos; siendo corroborado por los estudiantes con diversidad funcional, al destacar la participación de cada uno de los miembros principales del hogar, donde figuran los padres, madres y hermanos; al momento de proporcionarles ayuda en diferentes aspectos de la vida cotidiana.

Palabras clave: Funcionamiento parental; círculo familiar; diversidad funcional; percepción de apoyo; escala de funcionamiento parental.

\footnotetext{
Abstract

The aim of this research is to analyze the relationship of parental functioning, exercised by direct relatives or persons outside the family circle and the perception of support of students with functional diversity of the different schools of the
}

Technical University of Manabí. To study parental functioning, the parental functioning scale was used, aimed at 43 parents and to know the perception of support, an interview was applied to 74 students with functional diversity. As a result of the research it was found that within these families a good parenting function is maintained, for which the good communication and interaction and affective bonds are highlighted; being corroborated by students with functional diversity.

\section{Keywords}

Parental functioning; family circle; functional diversity; support perception; parental functioning scale.

\section{Introducción}

El rol y funcionamiento parental ejercido por los padres, cuidadores o familiares cercanos son puntos clave para el desempeño y desenvolvimiento de los hijos durante las actividades a realizar en toda su vida. Todos los deberes, derechos y normas que rigen dentro de un hogar y con cada uno de los integrantes de la misma; son elementos claves para conseguir la estabilidad y funcionamiento correcto dentro de un círculo familiar.

El termino discapacidad es producto de un concepto que ha ido evolucionado con el pasar del tiempo, primero haciendo referencia a la interacción entre las personas con deficiencias y barreras, que son resultado de la actitud de las personas que conforman su entorno y evitan el desarrollo pleno y activo una sociedad, que les 
brinda igualdad de condiciones con las personas de distintos medios sociales (OMS, 2001).

El concepto de discapacidad luego de ser considerado discriminador, cambia a ser característico; pasando a ser a ser vinculante y obligatorio, esto quiere decir que de tal manera que a las personas con algún tipo de discapacidad se las reconoce como autónomas y capaces de desenvolverse con normalidad en un ambiente con igualdad de oportunidades (Hernández, 2015).

Como lo indica la Organización Mundial de la Salud (OMS), en todo el mundo existe un aproximado de más de mil millones de personas que tienen alguna forma de discapacidad, casi 200 millones de ellas, experimentan algún tipo de dificultad considerables en su funcionamiento que les impide el correcto desenvolvimiento en sus distintas áreas (OMS, 2011).

Se ha notificado que en el Ecuador existe un total de 485.325 personas con diversidad funcional, previamente registradas en el registro nacional de discapacidad. Estas están divididas en cinco grandes grupos, tales como la discapacidad física con un $46.64 \%$, intelectual $22.32 \%$, auditiva $14.00 \%$, visual $11.06 \%$, psicológica $5.39 \%$ (Consejo Nacional para la Igualdad de Discapacidades, 2020). El Consejo Nacional Para la Igualdad de Discapacidades (CONADIS) refiere que la discapacidad es una condición que se puede dar en cualquier persona, sin importar estatus económico, social, sexo; envolviendo la interacción de una persona con sus dimensiones físicas o psicológicas. Estas incluyen un sinnúmero de dificultades que se pueden presentar desde problemas en la función o estructura del cuerpo, que pueden variar por limitaciones en algunas actividades físicas o realización de tareas diaria (CONADIS, 2020).

La presente investigación tiene como objetivo analizar la relación del funcionamiento parental, ejercido por familiares directos o personas no pertenecientes al círculo familiar y la percepción de apoyo de los estudiantes con diversidad funcional de las diferentes escuelas de la Universidad Técnica de Manabí.

\section{El funcionamiento parental}

El Premio Nobel de Economía Amartya Sen, argumenta la siguiente: "Una comprensión de las demandas morales y políticas de la discapacidad es importante, no sólo porque es un rasgo de la humanidad tan amplio y deficitario, sino también porque las consecuencias trágicas de la discapacidad pueden ser superadas sustancialmente con una ayuda social determinada e intervenciones creativas."

Las políticas que trabajen con esta habilidad pueden tener un dominio grande, incluyendo el aminoramiento de los efectos de la minusvalía, por un lado, y programas para prevenir el desarrollo de las discapacidades por el otro. Es extremadamente importante el poder comprender que la mayoría de las discapacidades son prevenibles, y mucho puede hacerse no sólo para disminuir el sufrimiento de la discapacidad, sino también para reducir la prevalencia de la discapacidad.

El vínculo de los padres dentro de un hogar es conocido como parentalidad, el cual es definido por Sallés y Ger (2011), la parentalidad hace referencia a las actividades desarrolladas por los padres y madres para cuidar y educar a sus hijos, al tiempo que promueven su socialización". De la múltiple variedad de los estilos de socialización, este se comienza a desarrollar desde muy temprana edad, con las figuras que los padres dan a conocer a sus hijos (Paredes, 2018).

Los padres, como principales entidades de adquisición de conocimiento para los hijos, son los responsables de la obtención de habilidades, recursos y competencias a emplearse en toda área y ambiente a desenvolver. De la misma manera, al conjunto de todas estas cualidades adquiridas se las denomina competencias parentales (Contreras (2013). Según Manjarrés \& Hederich (2018), relata que la educación parental se complejiza cuando los padres deben asumir la crianza de hijos con discapacidad, puesto que su papel es aún más importante en la generación de oportunidades que garanticen el desarrollo de sus potencialidades y su proyección personal.

Los padres o cuidadores con hijos que tengan diversidad funcional saben que desempeñan un papel de suma importancia en la vida de sus hijos. Como menciona Manjarres (2012), la familia está presente como referente en la vida social e individual de los seres humanos; por acción o por omisión se constituye en un punto de confluencia de sus prácticas, discursos; un campo de profunda ideologización e idealización”. Es por esta razón que la correcta crianza, el apoyo de cada uno de los miembros del hogar y de las personas que conforman su circulo social es de suma vitalidad para enseñar y formar bases de aprendizaje sobre sus dificultades y la manera correcta de llevarlas.

Como lo indica Sallés y Ger (2011), dentro del funcionamiento parental se encuentra una gran gama de tipos de familias; entre estas están las familias monoparentales, homoparentales, 
extensas, adoptivas; cada uno de estos tipos de familia, tiene su respectiva manera de proceder durante el proceso de formación de sus hijos y mucho más si dentro de la misma existe un hijo con diversidad funcional.

Dentro de la variedad de factores que conforman la familia, una de las principales características que predominan en los padres de hijos con diversidad funcional, es el miedo. Este surge de la escasa información que se tiene acerca de como debería ser su correcta crianza, juicios por parte de terceros, y culpabilidad por enfrentarse a sentimientos que no son aceptados. Por tales razones a diario se intenta que los hijos con discapacidad, lleven una vida normal, adquieranautonomía y desenvolvimiento en todos los aspectos y situaciones relacionadas con la vida diaria (Ureta, 2014).

El funcionamiento parental comienza mediante el proceso de duelo que deben pasar los padres o cuidadores al tener conocimiento de la discapacidad de sus hijos, por tal razón se debe reforzar la información acerca de las características, manifestaciones, potencialidades y dificultades que surgen a partir de la discapacidad; mediante esta información poder reflexionar sobre la forma en que como los padres pueden construir un ambiente adecuado para favorecer la convivencia familiar, el autoestima y por ende el sistema familiar (Fernández, 2012).

Como resultado de la situación presentada, se aspira que la familia sea un entorno en el cual se potencien las capacidades de las personas con diversidad funcional $y$ que se cuente con el apoyodurante el proceso de integración social. (Fantova, 2000). Por tal razón es fundamental que los padres sean conscientes que las dificultades presentadas por la persona con discapacidad no son un dato inalterable, sino por el contrario, estos si no llevan un correcto proceso pueden incrementar o reducir y que para todo ello la intervención familiar es fundamental.

En este sentido el tipo de apoyo brindado por los cuidadores puede representarse de diferentes tipos, por ejemplo: apoyo emocional que se muestra mediante la preocupación, empatía y expresiones de cariño- y/o apoyo instrumental; el cual está basado en dedicarles tiempo, proveerles de los recursos materiales necesarios y apoyarlos con sus problemas (Valdés, Carlos, Tanori, y Madrid 2016). Existen diferentes maneras y situaciones en las que cada uno de los miembros de la familia puede aportar al correcto desarrollo de la persona con diversidad funcional, teniendo como objetivo conseguir la autonomía en todas las actividades a realizar.

Dentro del funcionamiento parental y familiar, Araya (2007) afirma: "Las familias son las primeras en posicionarse frente al hecho de tener a una persona con discapacidad organizando distintas respuestas. Es por ello que las propias familias son el punto de arranque en la búsqueda de soluciones" (p.20). Por tal razón es fundamental crear procesos de comunicación que beneficien a cada uno de los miembros del hogar y de esta manera se facilite la expresión de sentimientos y poder proceder al cambio de actitudes en beneficio de tanto padres como hijos.

Internamente del mismo círculo familiar, se debe contar con soluciones y prestación de apoyo en los distintos ámbitos que se desenvuelva la persona con diversidad funcional, para que de esta manera se haga efectivo su derecho a una educación y una vida tan digna, plena, autónoma e independiente como sea posible (Fernández, 2012).

La funcionalidad parental y su relación con la percepción de apoyo de los estudiantes es de suma importancia conocer, debido a que todos los aspectos que la conforman junto con los integrantes de la familia, pueden llevar a tener un ambiente de crecimiento personal y mucho más cuando se cuenta con un integrante con diversidad funcional.

\section{Materiales y métodos}

La presente investigación, cuantitativa de alcance descriptivo, tiene como objetivo principal describir la incidencia del funcionamiento parental, el cual es ejercido por padres o cuidadores y conocer la percepción de apoyo de los estudiantes con diversidad funcional pertenecientes a las distintas escuelas de la Universidad Técnica de Manabí. Ante la respectiva búsqueda de informaciónpara la elaboración del presente artículo, no se ha encontrado precedentes de una investigación con el funcionamiento parental como variable independiente y los estudiantes con diversidad funcional como variable dependiente

Procedimiento: La obtención de información de la escala de funcionamiento parental, fue dividida en tres instancias, la primera en cual se convocóla asistencia decuidadores, responsables o padres de hijos con diversidad funcional pertenecientes a la Universidad Técnica de Manabípara la respectiva aplicación de la escala, que lleva como nombre Escala de Funcionamiento Parental (EFP),como segunda instancia los padres fueron visitados en sus respectivos hogares bajo su autorización y por 
último, algunos padres de familia asistieron a la Universidad para proceder con la entrevista $\mathrm{y}$ aplicación de la escala.

Para conocer la percepción de apoyo que tienen los estudiantes con diversidad funcional, de parte de padres, cuidadores o familiares se procedió a convocar a los alumnos en las respectivas instalaciones de la Universidad Técnica de Manabí, para la realización de la respectiva entrevista.

Participantes: Dentro de la investigación del funcionamiento parental, participaron 43 padres de hijos que forman parte del "Programa de intervenciones psicoeducativas sociales y clínicas, para el mejoramiento de la calidad de vida de los estudiantes con discapacidad de la Universidad Técnica de Manabí" y cuyos hijos presentan algún tipo de diversidad funcional.

Dentro del mismo programa se aplicó una encuesta a 74 estudiantes con diversidad funcional para conocer la percepción de apoyo recibida de parte de sus cuidadores o familiares cercanos.

Instrumentos: La recolección de información en la presente investigación se llevó a cabo utilizando dos métodos:

Para evaluar el funcionamiento parental en las familias con hijos con diversidad funcional se aplicó la Escala de Funcionamiento Parental (EFP). Esta escala está compuesta por seis componentes que estudian la funcionalidad de padres hacia hijos, valorando distintos aspectos como la comunicación, control conductual indulgente, control psicológico, control conductual rígido, vínculo afectivo y la subescala de coparentabilidad. (Arroyo, 2015). Esta herramienta de medida para padres, tiene como fin la pronta identificación de características disfuncionales vinculadas al síntoma de sus hijos.

Para obtener la información de la percepción de apoyo se utilizaron entrevistas transcritas aplicadas a los estudiantes de la Universidad Técnica de Manabí, pertenecientes al "Programa de intervenciones psicoeducativas sociales y clínicas para el mejoramiento de la calidad de vida de los estudiantes con discapacidad de la Universidad Técnica de Manabí”, en el periodo de febrero del 2019.

\section{Resultados}

En la aplicación de la Escala de Funcionamiento Parental a los padres o cuidadores de los estudiantes con diversidad funcionalque pertenecen a las diferentes escuelas de la Universidad Técnica de Manabí, se obtuvo información sobre diferentes áreas que conforman el ambiente familiar. Entre estos, resaltan seis áreas a estudiar:

- La Comunicación e interacción; donde destacan varias características, entre ellas la confianza hacia los padres para expresar sus sentimientos; tanto positivos como negativos, sin dejar a un lado la apertura para tratar temas o situaciones que les preocupen o sean de alta relevancia. De esta manera se reflejael interés hacia sus hijos, conociendo cada una de las necesidades que estos requieran o este interfiriendo en su correcto proceder de la vida diaria. Los padres o cuidadores manifestaron destinar parte de su tiempo de descanso para atender todo tipo de demandas presentadas y estas las justificaron con respuestas como "no quiero que nada malo les pase" "siento que es mi culpa que este así" "lo daría todo por su felicidad y bienestar".

- En el Control conductual indulgente se pudo constatar que, debido a la diversidad funcional de los hijos, los padres o cuidadores,al momento de poner límites, cumplen en gran cantidad estas normas, sin embargo, existen ocasiones donde no son respetadas y ceden ante las peticiones de los hijos como consecuencia del afecto que les tienen. Además de ello, los cuidadores constantemente recalcan las situaciones o acciones que no se estén realizando de manera eficaz.

- El Control psicológico y sobreprotección; donde padreso cuidadores de los estudiantes con diversidad funcional sienten que muchas veces agobian a sus hijos al estar siempre pendiente de ellos y en toda áreao situación que estén involucrados. Sin embargo, también dio como resultado que, constantementetratan de corregir la sobreprotección que tienen hacia sus hijos, dándoles el debido espacio personal.

- Control conductual rígido; los padres están totalmente convencidos de tener la razón en la mayoría de acciones o decisiones llevadas a cabo dentro del hogar, debido a que estas son tomadas en beneficio de cada uno de los integrantes del círculo familiar. La escala también dio como resultado que, los cuidadores nunca imponen fuertes castigos que impliquen 
algún tipo de violencia, con el fin de que sus hijos aprendan y no vuelvan a desobedecer. Por el contrario, suelen recurrir a ellos de una manera pasiva, comunicando las situaciones o acciones que no se están llevando de manera correcta.

- Vínculo afectivo; en este aspecto, dio como resultado que,los padres siempre muestran comprensión y empatía cuando su hijo está afligido, frustrado o decepcionado, además de esto sus expresiones de afecto son manifestadas mediante abrazos, besos y caricias a sus hijos. De esta manera tratan de alimentar el sentimiento de que estar con sus hijos, es disfrutar ellos.

- Subescala de acuerdo en la coparentabilidad; La pareja como tal, es decir, el padre y la madre, mencionaronser capaces de llevar en conjunto criterios que estén relacionados con normas, limites, castigos y todo aspecto involucrado directamente con sus hijos, el cual, como pareja, deseen transmitirles.

Para conocer la percepción de apoyo en los estudiantes con diversidad funcional de la Universidad Técnica de Manabí, se entrevistó a 74 estudiantes de diferentes escuelas de esta institución, de los cuales 37 eran hombres y 37 eran mujeres. Dentro del grupo de personas que mencionaron recibir apoyo, están: Mamá en un $47 \%$, papá $38 \%$, hermanos $6 \%$, abuelos $3 \%$ ninguno $3 \%$ y entre los rangos de amigos, hijos, cónyuge y padrinos están valorados en un $1 \%$ cada uno de estos componentes, gráfico 1.

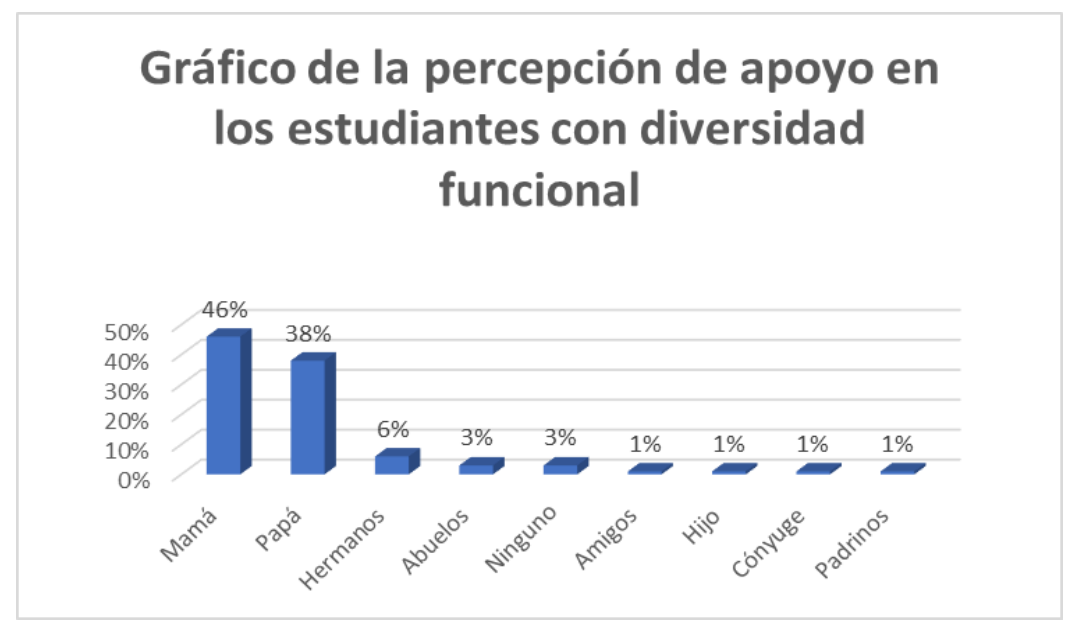

Gráfico 1. Percepción de apoyo en los estudiantes con diversidad funcional

Dentro del círculo familiar, conformado principalmente los padres, madres y hermanos, estando presentes al brindar apoyo en diferentes áreas, las cuales están divididas en apoyo físico, económico, educativo y emocional. Se obtuvo como resultado que el apoyo, tanto físico como económico, es brindado principalmente por el padre de familia, debido a que por el cabeza de familia es la persona encargada de llevar la económica dentro de los hogares; mientras que el apoyo emocional se expresa en mayor proporción por parte de la madre.

Durante la entrevista se encontró a madres que debido a la discapacidad de sus hijos tuvieron que dejar de realizar sus actividades laborales, pero a lo largo del tiempo y la dedicación hacia la correcta formación de sus hijos, obteniendo como resultado autonomía en todas las actividades que eran realizadas por ellos, incluyendo la persona con diversidad funcional, dichas madres decidieron retomar sus actividades laborales fuera de la casa, se observa en el gráfico 2 .

Cabe señalar que, aunque no predomina entre los mayores puntuados, está también el apoyo educativo y emocional brindado de parte de los hermanos. 


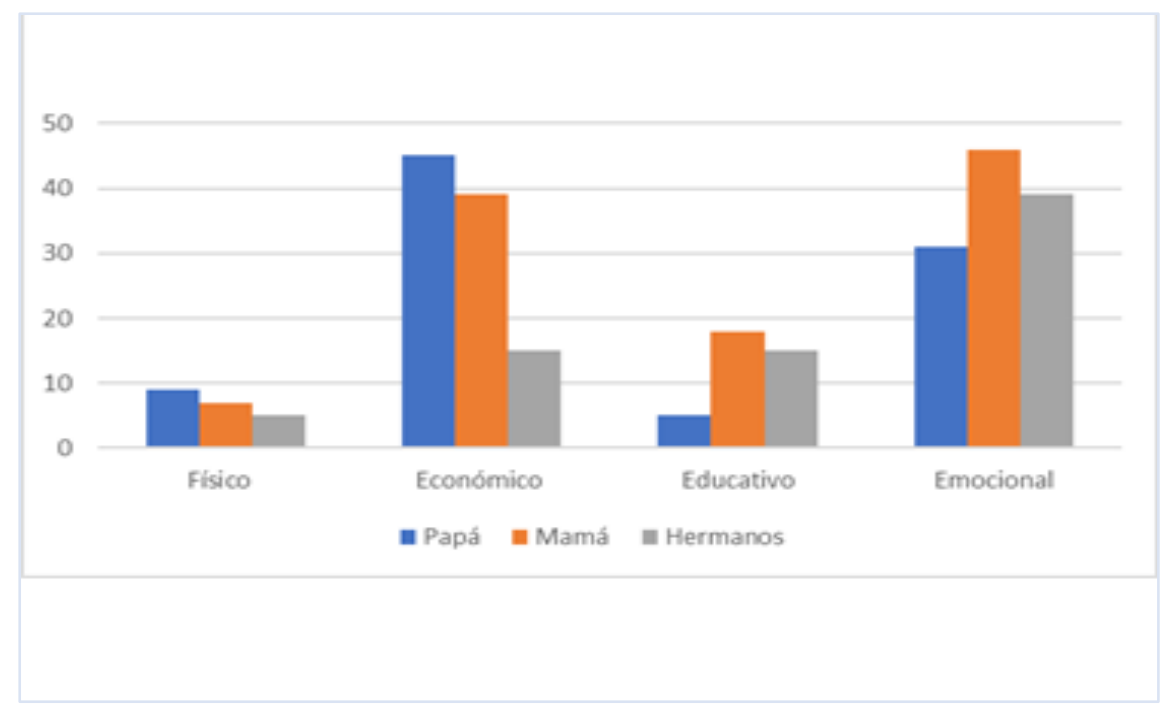

Gráfico 2. Percepción de apoyo de la familia a los estudiantes con diversidad funcional

\section{Discusión}

En este estudio se concuerda con otras investigaciones que han permitido conocer sus fortalezas y debilidades en esta materia. Entre las principales fortalezas destaca el liderazgo inclusivo, el diseño y la adaptación de actividades, y el buen ambiente de convivencia. Las debilidades apuntan a la escasa interacción de la escuela con su comunidad, así como a la nula colaboración con otros centros educativos del entorno (Azorín, 2017).

En lo concerniente al apoyo de la familia a los estudiantes con diversidad funcional la participación de las familias, se coincide con los autores Calvo, Verdugo, Amor (2016), señalan que para incrementar la participación familiar se han de planificar explícitamente actividades con esa finalidad, especificando con claridad los tipos posibles de participación y definiendo rotundamente el rol que corresponde desempeñar a las familias. En su investigación resaltan que los centros educativos buscan desarrollar nuevas formas de hacer y de ser para responder a los retos de la educación inclusiva. Contamos con modelos, herramientas y estrategias que favorecen una educación para todos, con experiencias positivas y con resultados beneficiosos y eficaces. En este estudio se concuerda con los autores citados anteriormente.

\section{Conclusiones}

A partir de todo lo expuesto en esta investigación, basada en conocer las diferentes situaciones relacionadas con el entorno familiar, el cual está compuesto por cuidadores o padres, madres e hijos y dentro del mismo forma parte un integrante con diversidad funcional o discapacidad. Específicamente al conocer situaciones relacionadas de padres hacia hijos y viceversa. Al estudiar la percepción de apoyo que tienen los hijos de parte de cada uno de los miembros que conforman el hogar, se puede constatar que, cada integrante aporta en distintos aspectos al miembro con diversidad funcional, dentro de estas, predominan cuatro características que son: apoyo físico, económico, afectivo y social, dando como resultado que los padres y las madres están más inmersos en el momento de darles apoyo físico, económico y emocional. Por lo tanto, los hermanos son las principales fuentes de apoyo afectivo y social.

Se evidenciómediante la aplicación de la escala de funcionamiento parental, que los padres o cuidadores de los hijos con diversidad funcional mantienen una buena comunicación entre ellos; dando como resultado que, dentro del hogar se pueda dar apertura a cualquier tema de interés o que esté afectando en algún ámbito de la vida del estudiante. Por otro lado, se mantienen buenos vínculos afectivos, debido aque los padres tienen claro el modelo de crianza que desean plasmar en sus hijos.

\section{Bibliografía}

Araya, S. (2007). De lo invisible y cotidiano. Familias y discapacidad . Revista Electronica "Actualidades investigativas en educación" , 1-21.

Arroyo, S. (Mayo de 2015). Escala de funcionamiento parental (EFP). Validación de un instrumento de medida para padres que sufren de Violencia Filio-Parental en 
sus familias. Obtenido de https://repositorio.comillas.edu/rest/bitstre ams/2646/retrieve

Azorín, C. (2018). Percepciones docentes sobre la atención a la diversidad: propuestas desde la práctica para la mejora de la inclusión educativa. ENSAYOS, Revista de la Facultad de Educación de Albacete, 33(1). Recuperado de http://www.revista.uclm.es/index.php/ensa yos

Calvo, M., Verdugo, M., y Amor, A. (2016). La Participación Familiar es un Requisito Imprescindible para una Escuela Inclusiva. Revista latinoamericana de educación inclusiva, 10(1), 99. 113. https://dx.doi.org/10.4067/S0718$\underline{73782016000100006}$

Consejo Nacional para la Igualdad de Discapacidades. (Febrero de 2020). Estadisticas de discapacidad. Obtenido de https://www.consejodiscapacidades.gob.ec /estadisticas-de-discapacidad/

Contreras, V. (2013). Competencia Parental y discapacidad intelectual: un estudio comparativo de familias. (Tesis doctoral en educación). Universidad Autónoma de Madrid, Madrid.

Fantova, F. (2000). Trabajar con las familias de las personas con discapacidades. Siglo Cero , 33-49.

Fernández, M. (2012). Descubriéndome como padre frente a la discapacidad de mi hijo. (Tesis de pregado). Universidad Veracruzana . Obtenido de Universidad Veracruzana .

Hernández, M. (2015). El concepto de discapacidad: de la enfermedad al enfoque de derechos . CES , 46-69.
Manjarres, D. (2012). Apoyo y fortalecimiento a familias para la crianza de niños con discapacidad. Horizontes Pedagógicos, 97118.

Manjarrés, D., \& Hederich, C. (2018). Estilos parentales en la discapacidad: examen de la evidencia empirica sobre un modelo. Revista Latinoamericana de Psicología, 187-200.

Naciones Unidas. (2008). Convención sobre los derechos de las personas con discapacidad y protocolo facultativo. 1-42.

OMS (2001). Clasificación Internacional del Funcionamiento, las Discapacidades y la Salud. Ginebra: OMS (CD-ROM, multilingüe. Versión 1.0).

Organización Mundial de la Salud . (2011). Informe mundial sobre la discapacidad.

Paredes, R. (2018). Estilo de socialización parental, funcionalidad familiar $\mathrm{y}$ agresividad en adolescentes en Trujillo (. Revista de investigación de estudiantes de Psicología "JANG", 70-94.

Sallés , C., \& Ger , S. (2011). Las competencias parentales en familias contemporáneas: descripción, promoción y evaluación. Educación Social, 23.

Ureta, P. d. (2014). Afrontamiento y estilos de crianza en padres de niños con discapacidad . Universidad Camilo José Cela , 29.

Valdés , A., Carlos , E., Tanori , J., \& Madrid , E. (2016). Relacion entre funcionamiento familiar, emociones morales y violencia entre estudiantes de primaria. Revista Mexicana de Investigación Educativa, 1093-1110. 